\title{
Oral and transdermal drug delivery systems: role of lipid-based lyotropic liquid crystals
}

\author{
This article was published in the following Dove Press journal: \\ Drug Design, Development and Therapy \\ 13 February 2017 \\ Number of times this article has been viewed
}

\author{
Rajan Rajabalaya \\ Muhammad Nuh Musa \\ Nurolaini Kifli \\ Sheba R David
}

PAPRSB Institute of Health Sciences, Universiti Brunei Darussalam,

Brunei Darussalam

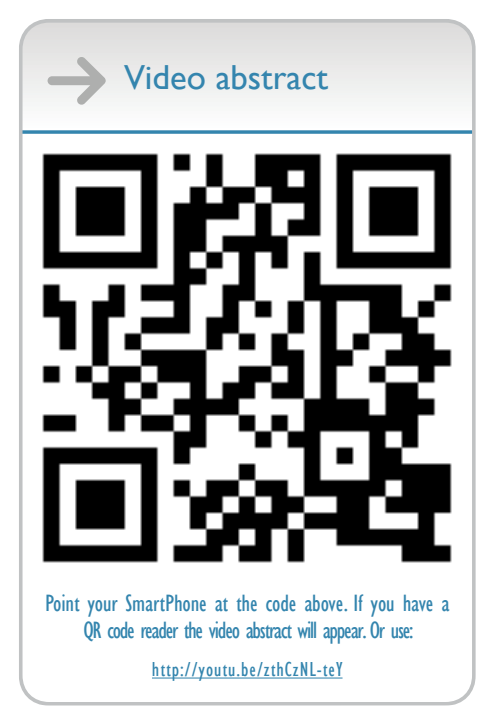

Correspondence: Sheba R David PAPRSB Institute of Health Sciences, Universiti Brunei Darussalam, Jalan Tungku Link, Gadong BEI4I0,

Brunei Darussalam

Tel +673246300I

Fax +673246 I08।

Email sdsheba@gmail.com

\begin{abstract}
Liquid crystal (LC) dosage forms, particularly those using lipid-based lyotropic LCs (LLCs), have generated considerable interest as potential drug delivery systems. LCs have the physical properties of liquids but retain some of the structural characteristics of crystalline solids. They are compatible with hydrophobic and hydrophilic compounds of many different classes and can protect even biologicals and nucleic acids from degradation. This review, focused on research conducted over the past 5 years, discusses the structural evaluation of LCs and their effects in drug formulations. The structural classification of LLCs into lamellar, hexagonal and micellar cubic phases is described. The structures of these phases are influenced by the addition of surfactants, which include a variety of nontoxic, biodegradable lipids; these also enhance drug solubility. LLC structure influences drug localization, particle size and viscosity, which, in turn, determine drug delivery properties. Through several specific examples, we describe the applications of LLCs in oral and topical drug formulations, the latter including transdermal and ocular delivery. In oral LLC formulations, micelle compositions and the resulting LLC structures can determine drug solubilization and stability as well as intestinal transport and absorption. Similarly, in topical LLC formulations, composition can influence whether the drug is retained in the skin or delivered transdermally. Owing to their enhancement of drug stability and promotion of controlled drug delivery, LLCs are becoming increasingly popular in pharmaceutical formulations.
\end{abstract}

Keywords: liquid crystal, drug delivery, controlled release, lyotropic, surfactants, drug localization

\section{Introduction}

Designing novel dosage forms to increase efficacy and stability of existing drugs is one of the formulation development processes performed in the pharmaceutical industry. Recently, liquid crystal (LC) dosage forms have generated substantial interest because they are applicable to both oil- and water-soluble compounds. ${ }^{1}$ LCs are substances that flow like liquids but maintain some of the structural characteristics of crystalline solids. LCs are classified into lyotropic LCs (LLCs) with amphiphilic mesogens and thermotropic LCs (TLCs) composed of mesogens that have a rigid component with one or two flexible aliphatic chains. Surfactants are important for the formation of LC phases, and they also enhance drug solubility by forming micelles. ${ }^{2}$ Micelles are formed because of the hydrophobic and/or hydrophilic nature of the molecules; micellar structures are often observed in LLCs. Once a certain concentration, known as the critical micelle concentration (CMC) or critical packing parameter (CPP), is reached, the molecules begin to twist into a sphere. ${ }^{3,4}$

In recent years, the use of LLCs as potential drug delivery systems has garnered significant attention, as they are able to provide a matrix with sustained drug release 
that can also protect nucleic acids and biologicals against both physical and chemical degradation. ${ }^{5}$ Many lipids that form LCs are nontoxic and biodegradable and are applicable to various routes of administration. ${ }^{6}$ In this review, we briefly introduce the structural evaluation of LCs and their effects in oral and topical formulations, including drug deposition and other factors. Our review is focused on research conducted over the past 5 years on the use of LLCs in drug delivery.

\section{Structural classification of LLCs}

The term "mesophase" is a common synonym for LCs and indicates their distinctive structure between a liquid and solid crystal phase. A liquid phase is fluid, while an ordered crystalline structure defines the solid state. ${ }^{3}$ The different mesophases of LLCs are lamellar, hexagonal and cubic (Figure 1). Water is often used as a solvent for LLCs; hydrophobic and/ or hydrophilic properties of the mesogens are important in determining the shape. ${ }^{7}$ If the solvent is water, the lamellar phase consists of the mesogens forming a bilayer with the hydrophilic heads as the outer layers and the hydrophobic tails as the inner layer; the lipid bilayers are arranged linearly with alternating water channels. However, if the solvent is organic, the orientation of the mesogens is reversed. ${ }^{8,9}$

The hexagonal phase usually consists of seven rod-like micelles arranged in a hexagonal three-dimensional lattice, that is, in cylinders. ${ }^{10}$ Normally, the hexagonal phase is obtained with water as solvent, while an organic solvent would yield the reverse form, known as the reverse hexagonal phase. A dispersed form of the hexagonal phase is known as a hexosome, a name trademarked by Camurus AB (Lund, Sweden) and registered under the US Patent and Trademark Office (USPTO). ${ }^{11}$

The cubic phase has two types, bicontinuous and discontinuous cubic (cubic micellar phase). The lipid bilayer in the bicontinuous cubic phase is arranged in a pattern of infinite periodic minimal surfaces (IPMSs). ${ }^{12,13}$ This is further divided into primitive $(\mathrm{Im} 3 \mathrm{~m})$, double diamond $(\mathrm{Pn} 3 \mathrm{~m})$ and gyroid (Ia3d) phases. The discontinuous cubic phase consists of the micelles arranged in a cubic lattice, which can be either a face-centered cubic (fcc) or a body-centered cubic (bcc; Figure 2A). Similar to the lamellar and hexagonal phases, there are reverse forms of both the bicontinuous and discontinuous cubic phases. ${ }^{11}$ Table 1 presents the compositions of some important LLC systems and their phases.

\section{Formulation of LLCs}

Nonionic surfactants have high interfacial activity because of the presence of both polar and nonpolar components. Compared with other surfactant categories, vesicles prepared from nonionic surfactants offer greater benefits with respect to stability, compatibility and toxicity. ${ }^{14}$ Formation of bilayer vesicles depends on the CPP (Figure 2B), the chemical structure of the components and the hydrophilic-lipophilic balance (HLB) of the surfactant. ${ }^{15}$ HLB values range from 0 to 18 ,

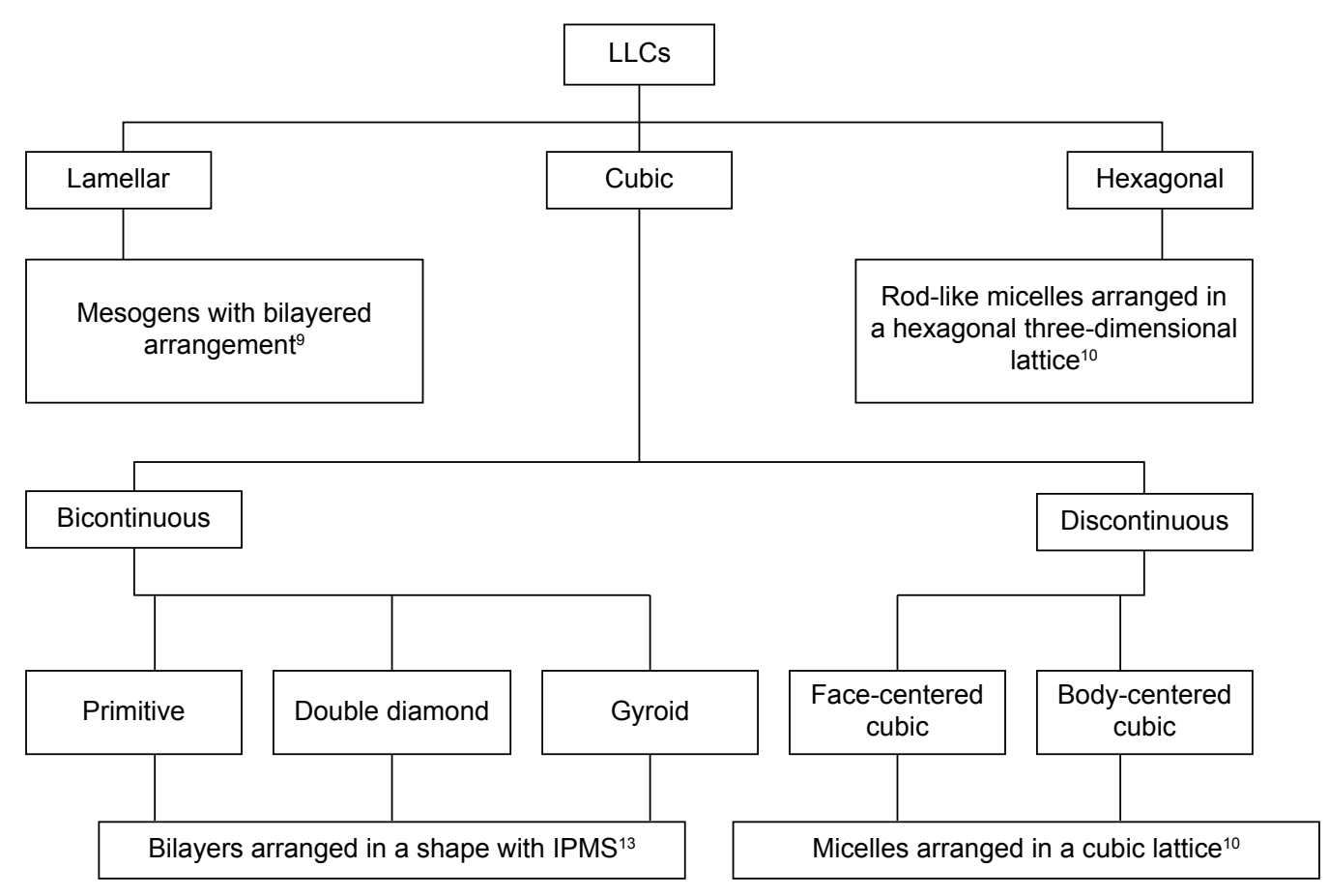

Figure I Classification of LLCs.

Abbreviations: LLC, lyotropic liquid crystal; IPMS, infinite periodic minimal surface. 
A

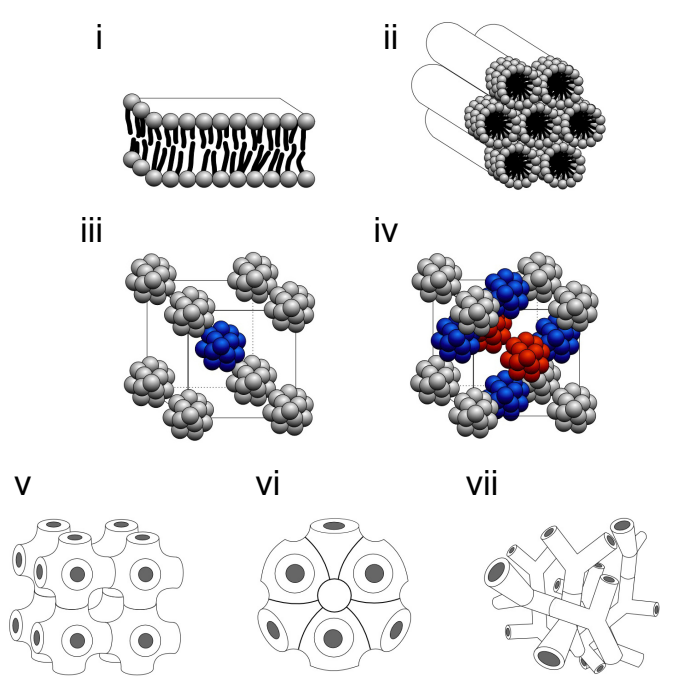

B

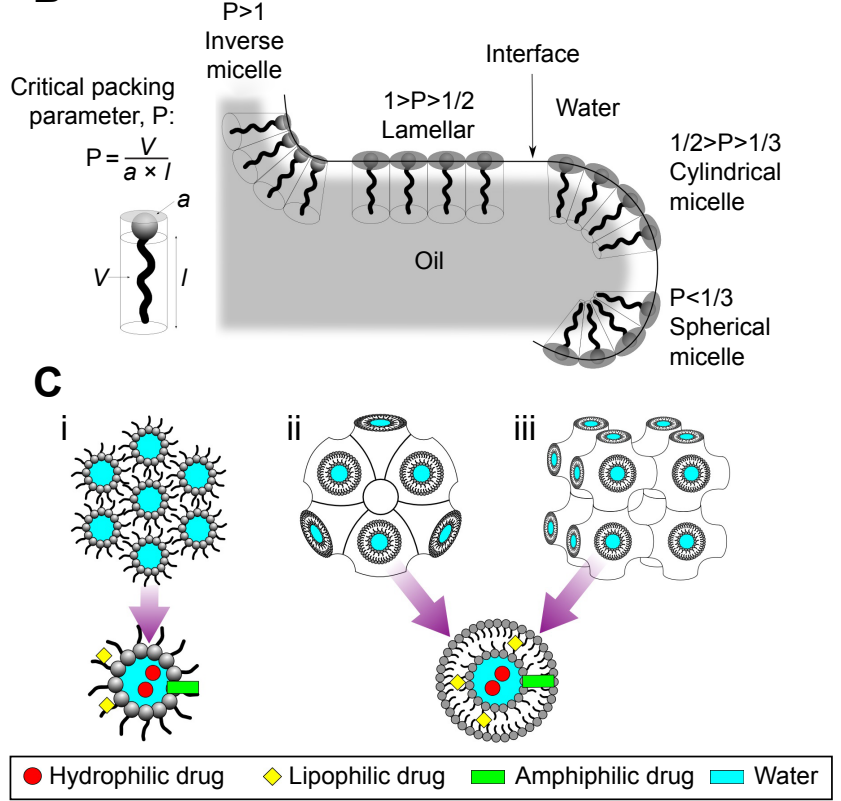

Figure 2 (A) The mesophases of LLC: i, lamellar phase; ii, hexagonal phase; iii, micellar cubic phase - bcc; iv, micellar cubic phase - fcc (different colors represent the threedimensional arrangement of the same molecules present in the micellar cubic phases); v, primitive bicontinuous cubic phase; vi, double diamond bicontinuous cubic phase; vii, gyroid bicontinuous cubic phase. (B) Schematic of self-assembly structures corresponding to CPP values. P stands for CPP values. $V$ is the hydrophobic tail volume, $a$ is the hydrophilic head group area and $I$ is the hydrophobic tail length. (C) Possible localization of drugs in the following: i, reverse hexagonal phase; ii, reverse bicontinuous cubic phase (double diamond); iii, reverse bicontinuous cubic phase (primitive).

Abbreviations: bcc, body-centered cubic; CPP, critical packing parameter; fcc, face-centered cubic; LLC, lyotropic liquid crystal.

where values $<10$ indicate lipid solubility while those $>10$ indicate water solubility. Based on their HLB values, the most effective surfactants can be selected and used to make the desired product. ${ }^{16}$ Entrapment efficiency (EE) is the ratio of weight of drug entrapped into a liquid crystalline carrier system to the total drug added. The $\mathrm{EE}$ and the in vitro release are very important physicochemical characteristics of liquid crystalline formulations. EE and HLB value determines drug EE of vesicles, where EE decreases along with the HLB value. ${ }^{17} \mathrm{EE}$ refers to the percentage of drug content within the vesicles out of the total drug in the dispersion. For surfactants with HLB values $>6$, adding lipids, such as cholesterol, is essential to form a bilayered vesicle. ${ }^{18}$ These bilayered repetitive structures are common in lamellar LLCs.

Table I Materials used to prepare LLCs

\begin{tabular}{lll}
\hline System & LC type & References \\
\hline P 407:MO/water & Cubic & 24 \\
P 407:GMO/water & Cubic & 22 \\
P 407:GMO/water & Cubic & 94 \\
P 407:MO:OA/water & Hexagonal & 24 \\
OG:PG:MO/water & Hexagonal & 20 \\
Tween 80:lipoid S-100/water & Lamellar & 95 \\
Span 80:Tween 80:sesame & Water-in-oil emulsion, & 88 \\
oil/polyvinyl alcohol & lamellar at aqueous phase & \\
\hline
\end{tabular}

Abbreviations: GMO, glyceryl monooleate; lipoid S-100, phosphatidylcholine from soybean; LC, liquid crystal; LLC, lyotropic LC; MO, monoolein; OA, oleic acid; OG, oleyl glycerate; P 407, poloxamer 407; PG, phytanyl glycerate.
A general procedure to make LLCs is to mix the oil phase, which can include monoolein (MO) and glyceryl monooleate (GMO) as well as surfactants, with the aqueous phase at a temperature of $40^{\circ} \mathrm{C}$, and then homogenize the mixture. ${ }^{12}$ The homogenization process is performed by either high-pressure homogenizers or manually rotating the tube around its axis, then shaking it using vortex and mechanical centrifugation. ${ }^{10}$ LLCs are viscous; thus, high-pressure homogenizers are usually preferred. ${ }^{19,20}$ After the homogenization process, a homogeneous, opalescent mixture is formed..$^{21,22}$ Many studies have described the process of incorporating lipophilic or hydrophobic drugs. ${ }^{21-24}$ Usually, drugs are loaded when the oil phase is in its molten state, before adding water.

Apart from the general procedure that is mentioned earlier, there are several other preparation methods: heat treatment and spray drying. ${ }^{3}$ In the heat treatment method, the gel formulation that was obtained from the general procedure was autoclaved to a temperature of $121^{\circ} \mathrm{C}$ for $\sim 15$ minutes followed by $\sim 5$ minutes of equilibration. Once these procedures were performed, LLC nanoparticles were obtained. In the spray-drying method, a powder form of each of the components was prepared, which would act as an LLC precursor. This precursor was then hydrated to form the LLC nanoparticles.

Formulation composition and preparation process are of paramount importance in determining the formation 
of liquid crystalline structure in emulsion. LCs are a combination of structure-forming lipids and aqueous phase, which are formed into a condensed state of matter by the special anisotropic surfactants such as GMO, MO and phytantriol (PT). When dispersed with high-energy process such as high-pressure homogenizer, the constituents arrange themselves to have an ordered long-range orientation network among molecules. The size range of emulsion is $1-5 \mu \mathrm{m}$, whereas LLCs are in submicron. The LLCs are anisotropic, and thus their formation can be ensured by observing them for their birefringence in crossed polarized light microscopy (CPLM) or small-angle $\mathrm{X}$-ray scattering (SAXS). ${ }^{25}$

\section{Drug localization and in vitro release behavior}

The release of drugs from LLC follows the Higuchi diffusion-controlled kinetics. ${ }^{26-28}$ Studies involving cubosomes and hexosomes have shown that there is an initial burst release of drugs before a sustained drug release is achieved. ${ }^{3}$ Stimuli, both endogenous and exogenous, may act as an on-off switch for the LLC. ${ }^{29}$ This was possible due to phase changes that took place as these stimuli acted on the LLC. ${ }^{30}$ The release behavior depends on the phase that the LLC assumes; the cubic phase usually releases its contents more rapidly than the hexagonal phase. ${ }^{30,31}$ Localization of drugs within the LC, as shown in Figure 2C, for cubic or hexagonal phases depends on the drug's solubility properties. Amphiphilic drugs would be localized along the oil-water interface, hydrophilic drugs within a water compartment or channel and hydrophobic drugs with the hydrophobic surfactant tails. ${ }^{3,32}$ Cubic bicontinuous structures, however, can carry large amounts of either hydrophobic or hydrophilic drugs. ${ }^{33}$ The hydrophilic head group size and the chain length of the nonionic surfactant affect the EE of the drug. Higher EE was observed in nonionic surfactants with stearyl (C18) chains compared with nonionic surfactants with lauryl (C12) chains. ${ }^{34}$ Surfactants belonging to Tween series contain large hydrophilic moiety and long alkyl chain, and when combined with cholesterol, they yield the highest EE with hydrophilic drugs. ${ }^{35}$ Based on an in vitro study on the cubic phase, the fraction of drug released and drug release kinetics were independent of the amount of drug loaded. ${ }^{36}$

\section{Phase behavior}

The phase behavior of LLC or mesophase behavior can be affected by several instances, such as the CPP of the
Table 2 Micelle shape corresponding to CPP values

\begin{tabular}{ll}
\hline CPP values & Micelle shape \\
\hline $\mathrm{CPP}<\mathrm{I} / 3$ & Spherical \\
$\mathrm{I} / 3<\mathrm{CPP}<\mathrm{I} / 2$ & Cylindrical \\
$\mathrm{I} / 2<\mathrm{CPP}<\mathrm{I}$ & Lamellar \\
$\mathrm{CPP}>\mathrm{I}$ & Inversed spherical \\
\hline
\end{tabular}

Abbreviation: CPP, critical packing parameter.

surfactants used and the introduction of another component in the formulation. ${ }^{3}$ The CPP value can then be used to predict the mesophase structure and the type of vesicle that are formed, which are shown in Figure 2B. Furthermore, it can also determine the mesophase behavior.

The formula to obtain the CPP value is as follows:

$$
\mathrm{P}=\frac{V}{a \times l}
$$

where $V$ is the hydrophobic tail volume, $a$ is the hydrophilic head group area and $l$ is the hydrophobic tail length. The CPP values that correspond to the mesophase and micellar structures are summarized in Table 2. In terms of the addition of another component to the formulation, a study has been carried out to investigate this. ${ }^{24}$ Initially, when MO and poloxamer 407 were used, a cubic phase was obtained. But when oleic acid was added, the cubic phase had changed to the hexagonal phase.

\section{Characterization of LCs}

It is important to characterize LCs, especially when they are used in drug delivery. Characterization identifies the phase into which the drug has been incorporated, an important parameter to predict drug release patterns, drug interaction with the targeted biological membrane and stability. Few structural parameters characterized are particle size, viscosity and morphology.

\section{Physiochemical characterization}

Particle size is an important parameter for the purpose of quality control, especially quality assurance, as particle size along with particle size distribution can influence the physical stability of vesicle dispersion. Generally, LCs are under $200 \mathrm{~nm}$ in size, but can reach up to $1 \mathrm{~mm}$; thus, photon correlation spectroscopy based on laser light scattering is commonly used as an appropriate method for measurement.

In a half logarithmic plot of the autocorrelation function, the slope of the graph enables calculation of the 
hydrodynamic radius, or particle size, according to the Stokes-Einstein equation:

$$
D_{h}=\frac{k_{B} T}{3 \pi \eta D_{t}}
$$

where $D_{h}$ is the hydrodynamic diameter, $D_{t}$ is the translational diffusion coefficient (calculated by dynamic light scattering), $k_{B}$ is Boltzmann's constant, $T$ is the thermodynamic temperature and $\eta$ is the dynamic viscosity.

The zeta average value can be determined using commercially available devices, and the values usually correspond to the hydrodynamic radius value. ${ }^{37}$ The particle size, which is the hydrodynamic size, when measured by dynamic light scattering is dependent on viscosity, which further relies on the temperature of the LLC.

Viscosity of LLCs varies according to their phase. The lamellar phase is the least viscous, while cubic phase is the most viscous among the LLC phases, whereas the hexagonal phase is more viscous than lamellar phase because of its higher water content. LCs that are too viscous are not suitable for drug delivery. Therefore, a less viscous form is preferred, as it would be expected to eventually transition into a more viscous form when in contact with aqueous media. ${ }^{38}$

Differential scanning calorimetry (DSC) is a relatively inexpensive and rapid method to measure heat capacities and enthalpy changes. As phase transition occurs, fluctuations in heat can be measured. However, aside from the LC phases, other structural information cannot be obtained with DSC..$^{39}$

The main purpose of SAXS is to visualize the X-ray scattering pattern produced by a material. With LCs, SAXS can be used to characterize phase behavior as well as structural detail, based on the scattering pattern. LC in SAXS produces a scattering two-dimensional pattern, which is then radially integrated to form a one-dimensional scattering profile. Based on Bragg's law, the scattered radiations satisfying the constructive condition would lead to strong intensities in the diffraction pattern. These intensities, known as Bragg peaks, can be used to identify the structure of the $\mathrm{LC}$, as each phase has a signature Bragg peak ratio (Table 3). ${ }^{40,41}$

A SAXS investigation was performed on a cubic phase dispersion to understand how the internal structure and size can be varied by controlling the poloxamer 407 ratio. $^{42}$ The presence and absence of poloxamer 407 in MO cubic phase produced primitive and double diamond structures, respectively. SAXS can also be used to further confirm the results from CPLM, as demonstrated in a study using a reverse hexagonal phase. ${ }^{43}$ Small-angle neutron scattering (SANS)
Table 3 Bragg peak ratios for LLCs

\begin{tabular}{|c|c|}
\hline Mesophase & Peak ratios \\
\hline Lamellar & $\mathrm{I}: 2: 3: 4$, etc \\
\hline Cubic micellar (fcc) & $\sqrt{3}: \sqrt{ } 4: \sqrt{ } 8: \sqrt{ } 11: \sqrt{ } 12$, etc \\
\hline Cubic micellar (bcc) & $\sqrt{ } 2: \sqrt{ } 4: \sqrt{ } 6: \sqrt{ } 8: \sqrt{ } 10$, etc \\
\hline Bicontinuous cubic $(\operatorname{Im} 3 \mathrm{~m})$ & $\sqrt{2}: \sqrt{ } 4: \sqrt{ } 6: \sqrt{ } 8$, etc \\
\hline Bicontinuous cubic (Pn3m) & $\sqrt{2}: \sqrt{3}: \sqrt{4}: \sqrt{6}: \sqrt{8}$, etc \\
\hline Bicontinuous cubic (la3d) & $\sqrt{ } 6: \sqrt{ } 8: \sqrt{ } \mid 4: \sqrt{ } 16: \sqrt{ } 18: \sqrt{ } 20$, etc \\
\hline Hexagonal & $\sqrt{3}: \sqrt{4}: \sqrt{7}: \sqrt{ } 12$, etc \\
\hline
\end{tabular}

Notes: $\operatorname{Im} 3 \mathrm{~m}$ indicates primitive phase, $\mathrm{Pn} 3 \mathrm{~m}$ indicates double diamond phase, and la3d indicates gyroid phase.

Abbreviations: bcc, body-centered cubic; fcc, face-centered cubic; LLC, lyotropic liquid crystal.

works on the same principle as SAXS, but it uses neutrons. SANS can be used to determine an LC structure based on its neutron scattering pattern. Although a neutron source is not always readily available, depending on its availability, SANS is sometimes used as a complement to SAXS.

\section{Morphological characterization}

LCs have anisotropic properties and produce bright double refraction or birefringence when viewed between two light polarizers arranged in a crossed position. ${ }^{44}$ In contrast, isotropic materials appear dark when viewed under the same settings. Therefore, CPLM is used as a standard tool to confirm the presence of an anisotropic material, in this case the LC ${ }^{45-47}$ Phase behavior characterization with CPLM of oleyl glycerate (OG) and GMO containing cinnarizine (CZ), for motion sickness or vertigo, with water and $0.1 \mathrm{~N} \mathrm{HCl}$ was studied. While GMO and $\mathrm{CZ}$ mixture formed an isotropic cubic phase, $\mathrm{OG}$ and $\mathrm{CZ}$ mixture formed a reverse hexagonal phase in contact with excess water ${ }^{48}$ However, analysis by CPLM is restricted to particle dimensions in the micron or submicron ranges for LCs; colloidal-based LLCs are in the nanoparticle size range thus can be resolved only by transmission electron microscopy (TEM).

Atomic force microscopy (AFM) analyzes the surface of a material and, by extension, can elucidate structural details and phase changes of LCs. ${ }^{22,49}$ In situ visualization of cubosomes and hexosomes was performed successfully using AFM, and structural modifications caused by addition of substances in situ were easily identified.$^{50}$ TEM offers high magnification enabling visualization of LC microstructure; however, TEM requires dehydrating samples by high vacuum process. During this process, LCs containing an aqueous portion undergo structural changes. Hence, a freeze-fracture technique is used to preserve the LC structure. In the freezefracture technique, two gold plates are used, where the sample is placed between them and then shock frozen with either liquid propane that is nitrogen cooled or slush nitrogen at $-196^{\circ} \mathrm{C}$ and $-210^{\circ} \mathrm{C}$, respectively. ${ }^{51}$ Environmental scanning electron 
microscopy (ESEM) is very similar in principle to scanning electron microscopy (SEM), but is more suitable for analyzing LCs because the dehydration step, which is mandatory in SEM, can be eliminated so that the LCs remain in their native state. ${ }^{52}$ This method produces three-dimensional images of the LCs, making it easier to identify the phases. ${ }^{53}$

\section{LLCs in formulations}

The use of LCs for sustained delivery of drugs has, in recent years, received much attention in the pharmaceutical literature. LLCs have been formulated to enhance the current drug delivery routes, such as oral, topical, intravenous and transdermal. Some of these LLC formulations are summarized in Table 4. Currently, LLC formulations for use in both oral and topical routes have been extensively carried out, which are described in the following sections.

\section{LLCs in oral formulations}

LLCs, such as the cubic phase dispersions, may prove advantageous for oral delivery for reasons, including 1) the structure could protect the drug against degradation in the gastrointestinal (GI) tract; 2) the lyotropic property of the cubic phase with a hydrophilic surface allows easy contact with the endothelial cell layer and can cross the water layer and 3) the ability for penetration across the endothelial cell membrane is enhanced. ${ }^{54}$

Hydrophobic drugs contained in LLCs are more stable and absorbed more easily than in more conventional formulations. However, the water and mucosal layers found within the small intestine can hinder the transport of hydrophobic drugs into endothelial cells. Secreted biliary lipids, such as bile salts (BSs), phospholipids (PL) and cholesterol, help to promote the formation of colloidal vesicles such as micelles, mixed micelles and emulsion droplets. ${ }^{55}$

These colloidal vesicles help in the solubilization of fatty acids, monoglycerides and triglycerides as well as poorly water-soluble drugs. In addition, secretion of biliary lipids may enhance drug solubility. ${ }^{56} \mathrm{BS}-\mathrm{PL}$ mixed micelles attenuated BS membrane damage, representing a significant advantage over BS simple micelles. ${ }^{57}$ According to the CPP

Table 4 Recent reports of liquid crystalline systems

\begin{tabular}{|c|c|c|c|c|c|}
\hline LC phase & $\begin{array}{l}\text { Lipid system } \\
\text { composition }\end{array}$ & $\begin{array}{l}\text { Drugs/bioactive } \\
\text { molecule }\end{array}$ & $\begin{array}{l}\text { Route of } \\
\text { administration }\end{array}$ & $\begin{array}{l}\text { Improvements to } \\
\text { drug delivery }\end{array}$ & References \\
\hline Lamellar & $\begin{array}{l}\text { Polyoxyethylene } 21 \\
\text { stearyl ether/oil/water }\end{array}$ & Itraconazole & Topical & Enhanced efficacy & 87 \\
\hline Cubic & $\begin{array}{l}\text { GMO/P 407/water and } \\
\text { PT/P 407/water }\end{array}$ & $C Z$ & Oral & Increased bioavailability & 94 \\
\hline Cubic & PT/P 407/water & Amphotericin B & Oral & Increased bioavailability & 54 \\
\hline Cubic & GMO/P 407/water & Cyclosporine A & Ocular & $\begin{array}{l}\text { Increased corneal } \\
\text { retention }\end{array}$ & 22 \\
\hline Cubic & $\begin{array}{l}\text { Isopropyl myristate/ } \\
\text { polyoxyethylated castor } \\
\text { oil/polyethylene glycol I00 }\end{array}$ & Paeonol & Transdermal & $\begin{array}{l}\text { Enhanced skin } \\
\text { permeability }\end{array}$ & 96 \\
\hline Cubic & MO/P 407/water & Tacrolimus & Intradermal & Drug retention & 24 \\
\hline Cubic & MO/P 407/water & Indomethacin & Topical & $\begin{array}{l}\text { Prolonged release } \\
\text { and anti-inflammatory } \\
\text { activity duration } \\
\text { increased }\end{array}$ & 23 \\
\hline Cubic & $\begin{array}{l}\text { Myverol I8-99/ } \\
\text { polysorbate 20/water }\end{array}$ & Celecoxib & Transdermal & $\begin{array}{l}\text { Improved skin } \\
\text { permeation }\end{array}$ & 76 \\
\hline Hexagonal & GMO/P 407/water & Vitamin K & Transdermal & $\begin{array}{l}\text { Increased transdermal } \\
\text { delivery }\end{array}$ & 77 \\
\hline Hexagonal & $\begin{array}{l}\text { GMO/oleic acid/F68/ } \\
\text { water }\end{array}$ & Progesterone & Oromucosal & $\begin{array}{l}\text { High EE, high } \\
\text { permeability and better } \\
\text { storage stability }\end{array}$ & 69 \\
\hline Hexagonal & $\begin{array}{l}\text { MO/P 407/oleic acid/ } \\
\text { water }\end{array}$ & Tacrolimus & Intradermal & Drug retention & 24 \\
\hline Hexagonal & $\begin{array}{l}\text { OG/phytanyl glycerate/ } \\
\text { myverol I8-99/water }\end{array}$ & Irinotecan & Intravenous & $\begin{array}{l}\text { Improved retention in } \\
\text { lactone form at near } \\
\text { neutral } \mathrm{pH}\end{array}$ & 20 \\
\hline Reverse hexagonal & P 407/MO/water & Vitamin $\mathrm{K}$ & Topical & Increased delivery & 77 \\
\hline
\end{tabular}

Abbreviations: CZ, cinnarizine; EE, entrapment efficiency; F-68, pluronic F-68; GMO, glyceryl monooleate; LC, liquid crystal; MO, monoolein; OG, oleyl glycerate; P 407, poloxamer 407; PT, phytantriol. 
concept, differential formations of either micelles or vesicles were possible using BS and PL mixtures, as indicated in Figure 2B. Moreover, as shown in Figure 3, BS-PL micelles promote increased oral bioavailability of poorly soluble drugs such as antipsychotic, phenothiazine and antifungal, amphotericin B. ${ }^{57}$ In the physiological environment of the GI tract, LLCs consist of an oil phase and a solubilized micellar phase during lipid digestion, which enhances drug solubility and bioavailability in the lumen. ${ }^{58}$

In the mucosal layer, as shown in Figure 3, the water layer acts as a barrier for lipid absorption, with the water layer preventing micelle formation. ${ }^{59}$ Mixed micelle formation plays an important role in the solubilization, transport and absorption of nonpolar bioactive components. ${ }^{60}$ The mixed micelles solubilize free fatty acids and transport the solubilized materials toward the surface of the enterocytes by traversing the mucosal layer. These solubilized materials are then absorbed at the enterocyte surface. ${ }^{48}$ Cubic phases prepared with CZ LCs were studied and showed improved sustained release and high drug payloads with in situ formation of the cubic phase. Sustained release of drugs from the cubic phase showed a low mean $\mathrm{C}_{\max }$ compared with the drug suspension. ${ }^{56}$ The researchers indicated that the release rates of $\mathrm{CZ}$ from the cubic $(\mathrm{Pn} 3 \mathrm{~m})$ liquid crystalline phase depended on the mesophase internal structure. The hydrophobic drug needs to cross the countless hydrophilic head domains systematically before being released via diffusion into the external continuous aqueous phase. ${ }^{61}$ Lee et $\mathrm{al}^{62}$ described the in vivo absorption behavior in rats of orally administered ${ }^{14} \mathrm{C}$-glucose, a drug release biomarker that is rapidly absorbed in LCs and in other formulations. The study results indicated that the nanostructure of these materials could modify the absorption kinetics of lipophobic drugs. GMO-based cubic phase LLCs of furosemide showed both sustained-release and mucoadhesive properties, along with increased gastric residence time and, consequently, enhanced bioavailability. ${ }^{63}$ Rapid diffusion and drug release occur with higher water content because of an increase in the channels available for release of hydrophilic drugs. ${ }^{41}$

Quercetin, a hydrophobic drug, is unstable in physiological medium when orally administered. An LC formulation of this drug $(5 \% \mathrm{w} / \mathrm{w})$ incorporating soy phosphatidylcholine (SPC) and glycerol dioleate (GDO) effectively solubilized quercetin in a nonaqueous SPC and GDO mixture resulting in its chemical stability. Interestingly, with a low SPC

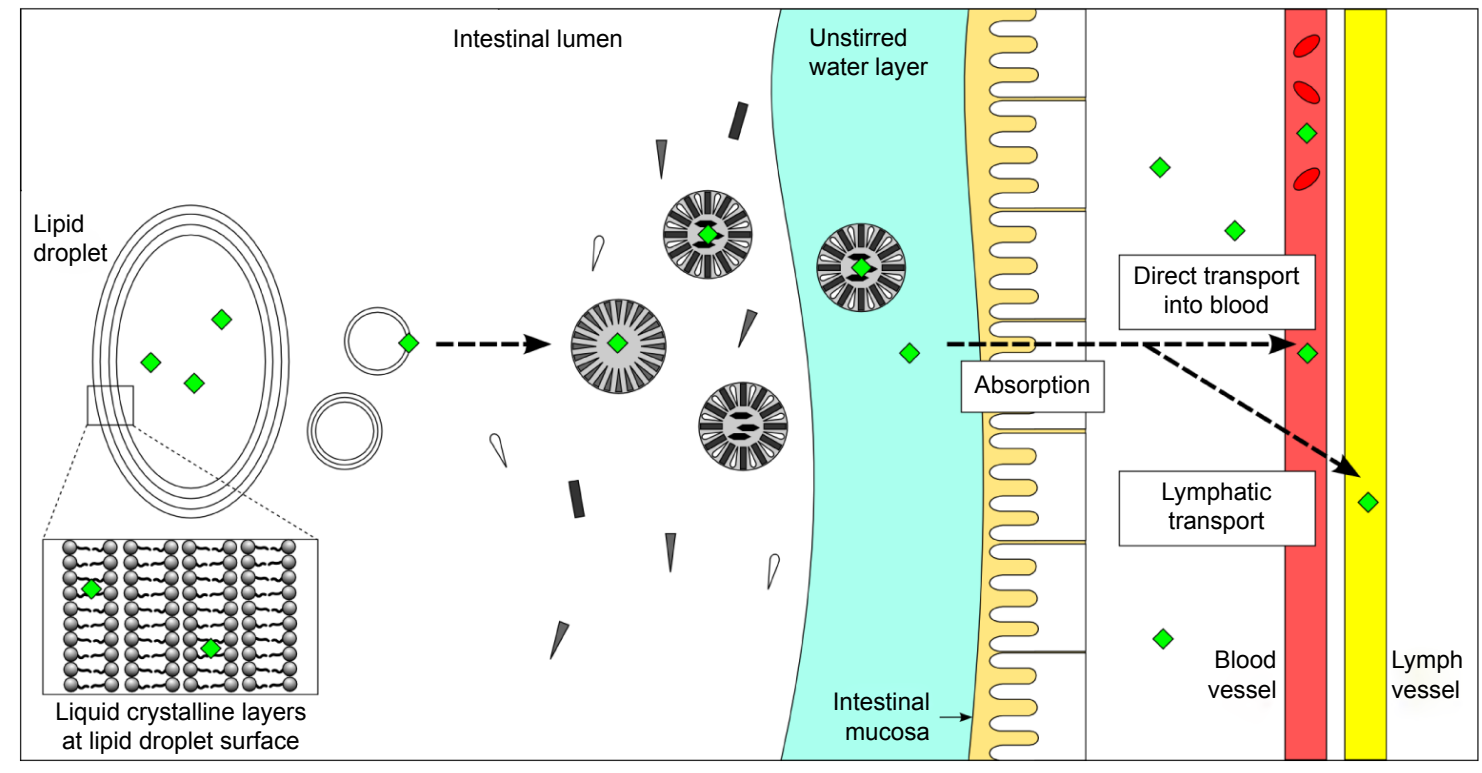

T Lipid digestion product (FA/MG/DG) | PL $\quad$ B BS $\quad$ Cholesterol $\diamond$ Drug

Figure 3 Drug solubilization within the small intestine.

Notes: The drugs are localized in lipid droplets possessing multiple layers of LCs on their surface. The layers are then shed from the droplet, forming small unilamellar or multilamellar vesicles. These vesicles are then solubilized into micelles and mixed micelles. Further solubilization would then promote lipid absorption across the intestinal mucosa.

Abbreviations: BS, bile salt; DG, diglycerol; FA, fatty acids; LC, liquid crystal; MG, monoglycerol; PL, phospholipid. 
content, the drug facilitated an increased unit cell dimension of the reversed micellar cubic phase ${ }^{64} \mathrm{LC}$ nanostructure led to better absorption kinetics of hydrophilic drugs and was found to enhance bioavailability by prolongation of gastric residence time. Hydrophobic drugs in LCs were more easily absorbed due to their increased stability and improved membrane penetration. An interesting phenomenon observed in LC nanoparticles was improved uptake mechanisms due to the formation of reversible "virtual pathways", which led to improved therapeutic efficacy. Another common advantage of LC is the reduction in systemic toxicity for antibiotics and chemotherapeutic drugs, while improving efficacy.

Delivery of siRNA was achieved using an LC formulation composed of monoglycerides, gene carrier polyethylenimines, propylene glycol and 0.1 M Tris buffer, pH 6.5. This mixture formed inverted-type liquid crystalline phases in contact with water. The presence of polyethylenimine influenced the formation of an siRNA complex formulation consisting of cubic and hexagonal phases in contact with additional water. This formulation showed sustained siRNA release for a week. ${ }^{65}$

\section{Drugs with poor oral bioavailability in LLC}

A formulation of cubic nanoparticles containing piperine loaded with 20(S)-protopanaxadiol, an anticancer drug with poor oral bioavailability, increased drug absorption. This bioavailability enhancement was caused by inhibition of drug metabolism, showing significantly increased apical to basolateral permeability across Caco-2 cell monolayers. ${ }^{53}$ A PT-based cubic phase embolic gelling solution is a promising carrier for the anticancer drug, hydroxycamptothecine, and was found to demonstrate sustained release by vascular embolization. Swelling of the PT matrices obeyed second-order kinetics and thus showed potential for clinical applications. ${ }^{66}$ LC systems were developed for the antibacterial agent poly(hexamethylene biguanide) hydrochloride with a GMO and water system, enabling delivery to buccal mucosa in a controlled drug release manner and improving its mucoadhesive properties. ${ }^{67}$ A lipid-based LC system increased the bioavailability not only for active synthetic pharmaceuticals and macromolecules but also for baicalein, a natural product from the root of Scutellaria baicalensis Georgi. ${ }^{68}$

An LC system consisting of linoleic acid and MO changed reversibly into a reverse columnar hexagonal phase from a primitive reverse bicontinuous cubic phase in the presence of water at $37^{\circ} \mathrm{C}$. Such system acts as a delivery vehicle for phloroglucinol, an antispasmodic hydrophilic drug.
The primitive cubic phase at neutral $\mathrm{pH}$ releases the drug faster than the reverse columnar hexagonal phase at $\mathrm{pH} 2$. Hence, this is suitable for administering drugs orally where the intended target is either in the intestinal or the colonic tracts. ${ }^{31}$ Salicylic acid LC systems were developed using GMO and water where drug loading, swelling rate and water content had no effect on the drug release, but were affected by the drug location. The release of lipophilic drugs that are incorporated within the lipid bilayers out into the water channels was identified as the rate-limiting step. Thus, the drug partitioning between the lipid phase and the aqueous phase affects the drug loading on the drug release profile. ${ }^{36}$

A study focusing on enhancement of antitumor efficacy and safety of orally administered doxorubicin and antioxidant coenzyme Q10 LC nanoparticles found that a reversible "virtual pathways" formation, along with better uptake mechanisms in the Caco-2 cells model, led to improved oral bioavailability. This combination improved the therapeutic antitumor efficacy $(\sim 1.76$-fold $)$ and decreased toxicity ( 4.5-fold) as compared with oral doxorubicin LC nanoparticle and intravenous doxorubicin, respectively. ${ }^{69}$ The combination of $\beta$-cyclodextrin and a GMO-based hexagonal diosgenin LC formulation enhanced the bioavailability of the poorly soluble diosgenin. ${ }^{70} \mathrm{~A}$ formulation of rifampicin encapsulated into cross-linked folate nanoparticle LCs showed sustained rifampicin release for 25 days. The formulation with the desired size range, $100-400 \mathrm{~nm}$, for tuberculosis drug delivery efficiently delivered the drug to macrophages without cytotoxicity. ${ }^{71}$

\section{Excipients used in LLC oral formulations}

There are many excipients used to improve the efficacy of the drug in LLC formulations. A study on lipid-based LCs showed that incorporation of nondigestible PT increased gastric retention of CZ LCs. These results demonstrated that there is a potential of achieving an optimal balance between sustained drug absorption and increasing plasma concentration $\left(\mathrm{C}_{\max }\right)$ over time, which then leads to enhanced oral bioavailability. The nondigestible nature of PT maintained the cubosome structure that contributed to sustained release and absorption of $\mathrm{CZ}$ in the stomach, leading to controlled plasma concentrations. ${ }^{72}$ Silica also stabilizes the GMO cubosomes but showed less enzymatic digestion than cubosomes stabilized with pluronic F127. This was attributed to the presence of a physical barrier by adsorbed silica nanoparticles. In addition, the excess dispersed silica nanoparticles adsorbed or scavenged the enzyme lipase. GMO cubosomes that were stabilized with silica were shown 
to be able to solubilize $\mathrm{CZ}$ at a greater capacity compared with pure drug suspensions when placed under simulated intestinal digestion conditions. ${ }^{73}$ Nguyen et $\mathrm{al}^{74}$ developed reverse hexagonal LCs of CZ, a poorly water-soluble drug, using selachyl alcohol. This formulation showed significantly enhanced oral bioavailability compared with the control suspension. Significantly, sustained absorption of CZ from the hexagonal systems employing selachyl alcohol, a nondigestible lipid, led to a longer $\mathrm{T}_{\max }$. Lipid-based LC materials developed for the PT cubic phase with sucrose stearate, a hydration-modulating agent, increased the water channel size incrementally when excess water is present. This study demonstrated that loading and release of macromolecules, for example, antibodies, can be controlled. ${ }^{75}$

OG was less susceptible to pancreatic lipase hydrolysis than GMO. An aqueous solution of $\mathrm{CZ}$ was administered orally and compared with GMO and OG solutions. Initial studies indicated that the OG formulation, unlike GMO, had the highest plasma concentration after $30 \mathrm{~h}$ of dosing. Subsequent studies confirmed the superiority of OG including significantly increased relative oral bioavailability, in a plasma sample taken $120 \mathrm{~h}$ after dosing. This confirmed that lipids that form LC structures in highly hydrated conditions have potential uses in sustained-release oral delivery systems because of their nondigestible nature. ${ }^{48}$ The alkyl glycerates in OG formed a reverse hexagonal phase in excess water in oral ingestion studies, allowing drug diffusion through hydrophobic alkyl glycerate chains. This structure was responsible for the controlled release of the drug from the liquid crystalline matrix..$^{20,45}$ The drug diffusion-controlled release rates, which are only available in the rich polymorphism of the LLC systems, follow the pattern, $\mathrm{D}_{\text {bicontinuous_cubic }} \geq \mathrm{D}_{\text {hex }} \geq \mathrm{D}_{\text {micellar_cubic }}{ }^{30}$

The nondigestible excipients demonstrated increased retention time in the appropriate place, leading to sustained absorption and furthermore to increasing plasma concentration and bioavailability as well as leading to a longer $\mathrm{T}_{\max }$. Likewise, hydration of modulating agents led to improved loading and release of drugs due to the incremental increase, in water channel, proportional to the presence of water level. Excipients that had reduced enzymatic digestion or scavenged the enzyme by offering a physical barrier or adsorbed the enzymes were effective in producing a longer $\mathrm{T}_{\max }$.

Ingredients in LLCs that made the formulation less susceptible to enzymatic hydrolysis increased the plasma concentration increasing their relative oral bioavailability. Moreover, it was observed that lipid formulations formed in highly hydrated conditions produced rich polymorphism.
These formulations allow consistent and efficient drug diffusion thus are responsible for controlled release.

Furthermore, high drug payloads observed with LLCs were responsible for sustained release and increased drug absorption. Increased inhibition of drug metabolism, due to the formation of cubic nanoparticles led to increased apical to basolateral permeability. LLCs not only exhibited sustained release by vascular embolization but also improved mucoadhesive properties. In addition, $\mathrm{pH}$-responsive LLCs reversibly switch structural and physical properties and were found to be useful for colon drug delivery.

\section{LLCs in topical formulations}

The stratum corneum (SC), the outermost layer of the epidermis, can act as a barrier for both topical and transdermal drugs. Some drug substances were able to form mesophases either together with LLCs or alone. Drug substances that form LLCs may interact with mesomorphous vehicles and liquid crystalline structures that are present in human tissues. ${ }^{37}$

The use of LLCs to deliver drugs has made it possible for the drug to be localized within the SC. One example drug that was incorporated in LLCs is celecoxib. In this case, the drug is loaded into the cubic phase and as the formulation is applied topically, the drug becomes localized within the SC, with some permeation through the skin. ${ }^{76}$ Similar behavior is also observed when vitamin $\mathrm{K}$ is delivered using the hexagonal phase, where minimal transdermal delivery also occurs, thus avoiding the side effects. ${ }^{77}$ In addition to this, vitamin $\mathrm{K}$ that is incorporated into either a GMO and water-based hexagonal-phase gel or GMO and F127-based hexosomes delivered 2-3.7 times more across the SC than a control formulation using petroleum jelly. ${ }^{78}$

Drug permeation has also enhanced when LLCs are used topically. Surfactant-based transdermal delivery of nonsteroidal anti-inflammatory drug (NSAID) flurbiprofen vesicles prepared by a film rehydration and extrusion method using L-595, sorbitan monolaurate and sodium dodecyl sulfate, showed the highest drug permeation compared with other formulations when all were tested on animal skin. Vesicle structure was maintained in the formulation, with retained and enhanced hydration as well as increased flurbiprofen transport. By polarized microscopy, vesicles showed optical isotropy because the formulation changed toward a liquid crystalline hexagonal and lamellar state on evaporation of water. This correlated with the decreased flurbiprofen permeation after $8 \mathrm{~h}$ of application. ${ }^{79}$

Drug permeation was also enhanced by $106 \%$ using retinyl palmitate LCs compared with retinyl palmitate emulsion. 
This was attributed to the similarity of the structures of LCs and SC leading to easy distribution as well as to the efficient hydration effects of LCs, as compared with the emulsion. ${ }^{80,81}$ The NSAIDs, including diclofenac diethylamine or ibuprofen lysinate, are organic acids with amphiphilic properties and, at room or body temperature, form LLC mesophases in aqueous solution. ${ }^{82}$

Incorporation of anxiolytic baicalin into a GMO cubic phase gel with diethylene glycol monoethyl ether resulted in a higher transdermal flux than baicalin incorporated into a carbopol-based hydrogel. Moreover, the cubic phase gel is more viscous compared with lamellar phase gel. The cubic phase forms a biological membrane-like structure that was suggested to promote a strong bioadhesive property to the skin, thus enabling the fusion of GMO with the lipophilic SC layer where baicalin was then released from the curved bicontinuous lipid bilayer. On the other hand, the formulation of an alkaloid, extracted from Tabernaemontana divaricata, which consisted of Zingiber cassumunar oil, Triton X-114, ethanol and water, as an LC system without microemulsion, enhanced the extract's transdermal permeation. In this study, the enhancement was hypothesized to be caused by the presence of interlamellar water in the LC phase which acts as a reservoir for controlled cutaneous hydration. ${ }^{83}$

Ringing gels are cubic LC-based system of optically transparent surfactant gels that has audible range resonance effects. ${ }^{37}$ Such gels have been used for topical NSAID formulations, which are commercially available, Dolgit Mikrogel (ibuprofen) and Contrheuma Gel Forte N (bornyl salicylate, ethyl salicylate and methyl nicotinate) ringing gels, to name a few. It is believed that the cubic phase may interact with the SC structure, leading to the formation of a cubosomal monolein-SC lipid mixture and a cubosome depot, as shown in Figure 4, from which the drug could be released in a controlled fashion. ${ }^{84}$ The cubic phase can also form a biological membrane-like structure that was suggested to promote a strong bioadhesive property to the skin. ${ }^{85}$

In addition, these gels contain high surfactant concentration, which can cause the gels to interact with microstructure of the SC lipids, thus increasing drug permeability. An example of a surfactant that can interact with the SC lipids is polyoxyethylene 20 cetyl alcohol. This surfactant forms the LC system when coming into contact with water and oil. ${ }^{86}$ GMO is another surfactant that can interact with the SC. An LC formulation consisted of GMO and poloxamer 407 loaded with cyclosporine A, used for immunosuppression, has shown this characteristic, where the GMO, an absorption enhancer, has promoted reversible disruption of the lamellar lipid bilayer in the SC, thus boosting the intercellular lipid fluidity. Moreover, cyclosporine A formulated with GMO and poloxamer 407 LC nanoparticles prepared for ocular drug delivery system caused decreased ocular irritancy and improved corneal penetration, when compared with a control cyclosporine A formulation. This low-irritant vehicle in LC nanoparticles may have the potential to deliver drug via the ocular route effectively. ${ }^{22}$

LLCs with itraconazole were developed using a system of polyoxyethylene stearyl ether, oil and water. Compared with a control formulation, these produced higher antimicrobial

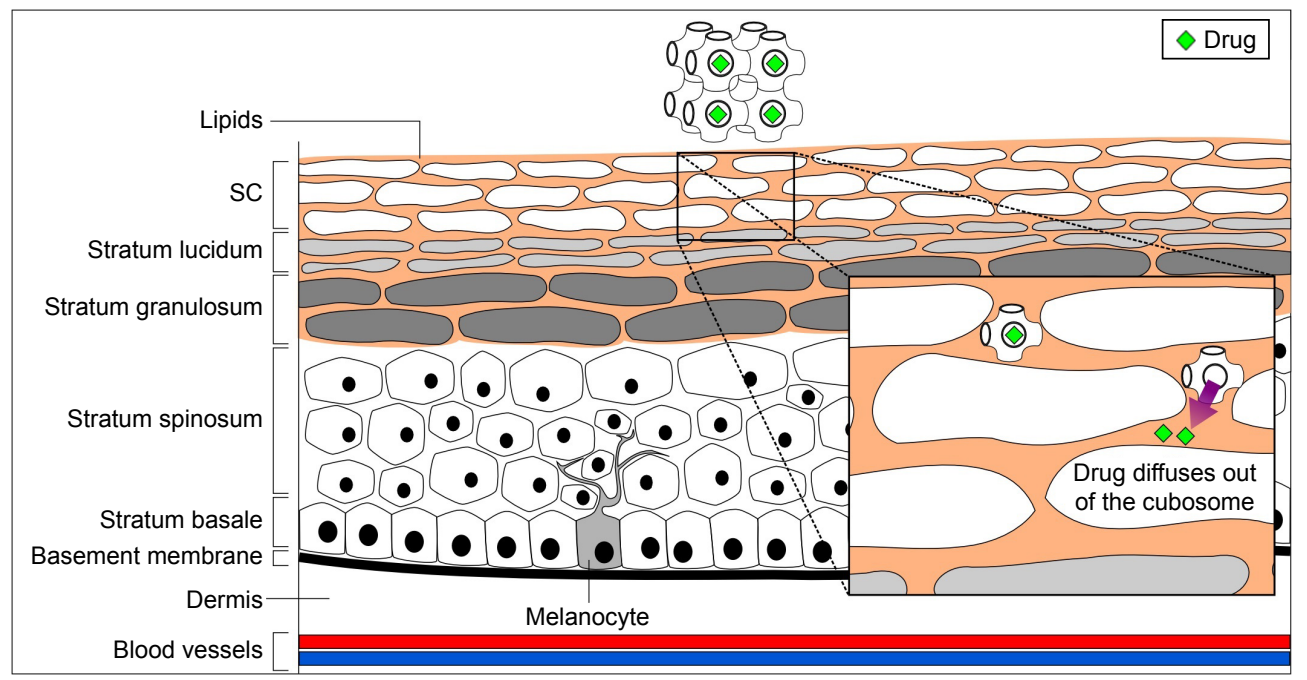

Figure 4 Transdermal drug delivery using LCs.

Note: Representative primitive bicontinuous cubic phase is shown.

Abbreviations: LC, liquid crystal; SC, stratum corneum. 
efficacy and inhibition against Candida albicans. ${ }^{87}$ In one study, novel organogel-based LLCs were prepared and investigated as controlled release matrices. The LLC gels were prepared using mixtures of Span 80-Tween 80 $(1: 2 \mathrm{w} / \mathrm{w})$ and an aqueous solution of polyvinyl alcohol $(10 \%$ $\mathrm{w} / \mathrm{w}$ ) and sesame oil. The biocompatibility of these gels, loaded with antibiotic metronidazole, was tested in human keratinocytes. Polarized micrographs showed formation of lamellar structures within the aqueous phase of the matrices. Metronidazole release from the gels was diffusion mediated without any cytotoxic effects on human keratinocytes and with good antimicrobial efficacy against Escherichia coli. ${ }^{88}$

In summary, drugs in LLC interact with tissue and localize in $\mathrm{SC}$, which results in controlled drug release ensuring minimal systemic delivery, consequently avoiding side effects. Correspondingly, tissue interaction resulted in decreased irritancy and improved penetration in cornea, thus accomplishing a potential ocular delivery system due to increased intercellular lipid fluidity boosted by absorption enhancers. Moreover, LC and SC structural similarity improved drug distribution and tissue hydration.

Therefore, changing the excipients of LC and its structure was found to improve drug penetration by increased transdermal permeability, which is a desired feature for antibiotic therapies. Tissue hydration is an advantage due to the presence of water in $\mathrm{LC}$ which acts as a reservoir. Similar to oral systems, topical formulations also showed less cytotoxic effects with higher therapeutic efficacy. Moreover, the maintenance of vesicle structures was proportional to drug transport. Transdermal flux, of LC formulation, was found to be higher and retained strong bioadhesive nature to skin.

\section{Challenges of using LLCs for drug delivery}

There are few challenges in the use of LCs for clinical therapeutic use. The main challenges and solutions given by researchers to overcome these are explained in the following paragraphs.

One of the current challenges in the use of LCs is the effect of their structure on prolonged storage, especially at low temperatures. Several biopharmaceuticals often use $4^{\circ} \mathrm{C}$ as a condition for storage purposes. A study involving a cubic phase swollen with water was shown to be stable at temperatures down to $0^{\circ} \mathrm{C} .{ }^{89}$ In addition, such phase was reversible. For LLC in an emulsion form, no significant changes were observed within the first 12 months of storage..$^{90}$ However, after 18 months, the structure starts to decompose. In this study, no drugs were used, so it would be interesting to know whether the presence of drug may affect the decomposition process. A study that used irinotecan in OG/oleic acid formulation showed that lower $\mathrm{pH}$ may increase the storage time of the LC structure, in this case, the hexosome. ${ }^{20}$

LLCs exist spontaneously when the amphiphilic lipids get into contact with excess water, forming ordered, liquid crystalline structures. In addition, these structures are thermodynamically stable. However, the main challenge is whether the LLCs would retain their nanostructure and properties when temperature is altered. This was demonstrated in a study in which such system formed the cubic phase at lower temperatures, but it transitioned into the hexagonal phase when the temperature increased, despite the excess water conditions. ${ }^{29,30}$ These transitions were reduced by adding oleic acid in GMO LLC systems and vitamin E acetate in PT LLC systems. ${ }^{91}$ Therefore, conditions such as temperature and $\mathrm{pH}$ may be manipulated to prolong the storage time.

As mentioned earlier, stimuli such as temperature and $\mathrm{pH}$ may act as an on-off switch. However, only these two have been studied broadly, despite other stimuli such as light, magnetic field and electric field are available. ${ }^{29-31}$ These other stimuli may be utilized but identifying which component to be used in the formulation that responds to these stimuli may prove to be a challenge. In addition, the components should not pose a risk or hazard when used in vivo.

Despite the usefulness of initial burst release, it may also cause some negative effects. ${ }^{26}$ Some of these effects include local or systemic toxicity, short release profile and wastage of drug. Therefore, this poses a challenge of using LCs in drug delivery, especially drugs that are to be used for long term. A model study managed to minimize this issue by selective alkylation of the drug. ${ }^{92}$ In this study, tryptophan was used and released from the cubic phase with different geometries. The study concluded that increasing the alkyl chain length may prolong the release, and in addition the release rate was also affected by changing the alkyl linkage type and its charge.

The amount of drug that can be loaded into an LLC may be affected by the amount of surfactants and cosolvents used. However, using high amounts of these two components may prove to be harmful and has caused a limited number of formulations that can be used. ${ }^{93}$ LLC with high drug capacity is an important parameter for long-term use formulation. In addition, it also ensures that there is still drug remaining even after the initial burst release for subsequent use. Therefore, the main challenge is to produce an LLC drug carrier that has higher drug load but uses the least amount of surfactants and cosolvents. 


\section{Conclusion}

This review on lipid-based LCs primarily discussed the current status of their applications in oral, topical and transdermal drug delivery systems. The structural classification of LLCs into lamellar, hexagonal and micellar cubic phases was described. LLC structure influences drug localization, particle size and viscosity, which, in turn, determine drug delivery properties. In oral LLC formulations, micelle compositions and the resulting LLC structures can determine drug solubilization and stability as well as intestinal transport and absorption. Similarly, in topical LLC formulations, composition can influence whether the drug is retained in the skin or delivered transdermally. Because of their enhancement of drug stability and promotion of controlled drug delivery, LLCs are becoming increasingly popular in pharmaceutical formulations. However, the pharmaceutical commercialization of these drug delivery systems, while potentially exciting, has challenges including industrial scalability and the control of lipid phase behaviors. The future of lipid-based liquid crystalline drug delivery systems, however, is of great interest in both the academic and industrial sectors.

\section{Highlights}

- Utilizing lyotropic liquid crystals (LLCs) to deliver drugs orally and via the skin

- Drugs are localized in LLCs based on their hydrophobicity

- Surfactants can influence the structure of LLCs

- Controlled release of drugs can be achieved using LLCs.

\section{Acknowledgments}

This project was supported by the University Research Grant of Universiti Brunei Darussalam, grant no UBD/PNC2/2/ RG/1(317).

\section{Disclosure}

The authors report no conflicts of interest in this work.

\section{References}

1. Hitesh J, Rushikesh G, Gauri J, Jagruti M, Nirali T. Liquid crystal as accelerant in drug absorption from topical formulations. Int Res JPharm. 2011;2(4):86-89.

2. Sekhon BS. Surfactants: pharmaceutical and medicinal aspects. JPharm Technol Res Manag. 2013;1:11-36.

3. Guo C, Wang J, Cao F, Lee RJ, Zhai G. Lyotropic liquid crystal systems in drug delivery. Drug Discov Today. 2010;15(23-24):1032-1040.

4. Nagarajan R, Ruckenstein E. Critical micelle concentration: a transition point for micellar size distribution. $J$ Colloid Interface Sci. 1977;60(2):221-231.
5. Mohammady SZ, Pouzot M, Mezzenga R. Oleoylethanolamide-based lyotropic liquid crystals as vehicles for delivery of amino acids in aqueous environment. Biophys J. 2009;96(4):1537-1546.

6. Ganem-Quintanar A, Quintanar-Guerrero D, Buri P. Monoolein: a review of the pharmaceutical applications. Drug Dev Ind Pharm. 2000;26(8): 809-820.

7. Kovarova A, Svetlik S, Kozmik V, et al. Unusual polymorphism in new bent-shaped liquid crystals based on biphenyl as a central molecular core. Beilstein J Org Chem. 2014;10:794-807.

8. Lusiana N, Muller-Goymann CC. Preparation, characterization, and in vitro permeation study of terbinafine $\mathrm{HCl}$ in poloxamer 407-based thermogelling formulation for topical application. AAPS PharmSciTech. 2011;12(2):496-506.

9. Lindblom G, Larsson K, Johansson L, Fontell K, Forsen S. The cubic phase of monoglyceride-water systems. Arguments for a structure based upon lamellar bilayer units. J Am Chem Soc. 1979;101(19): $5465-5470$.

10. Figueirdo Neto AM, Salinas SRA. The Physics of a Lyotropic Liquid Crystals. New York, NY: Oxford University Press; 2005.

11. Rosa M, Infante MR, Miguel MDG, Lindman B. Spontaneous formation of vesicles and dispersed cubic and hexagonal particles in amino acid-based catanionic surfactant systems. Langmuir. 2006;22(13): 5588-5596.

12. Spicer PT, Hayden KL, Lynch ML, Ofori-Boateng A, Burns JL. Novel process for producing cubic liquid crystalline nanoparticles (cubosomes). Langmuir. 2001;17(19):5748-5756.

13. Fontell K. Cubic phases in surfactant and surfactant-like lipid systems. Colloid Polym Sci. 1990;268(3):264-285.

14. Jiao J. Polyoxyethylated nonionic surfactants and their applications in topical ocular drug delivery. Adv Drug Deliv Rev. 2008;60(15): 1663-1673.

15. Ghanbarzadeh S, Khorrami A, Arami S. Nonionic surfactant-based vesicular system for transdermal drug delivery. Drug Deliv. 2014;22(8):1-7.

16. Fernandes CP, Mascarenhas MP, Zibetti FM, et al. HLB value, an important parameter for the development of essential oil phytopharmaceuticals. Rev Bras Farmacogn. 2013;23(1):108-114.

17. Shahiwala A, Misra A. Studies in topical application of niosomally entrapped nimesulide. J Pharm Pharm Sci. 2002;5(3):220-225.

18. Paecharoenchai O, Teng L, Yung BC, Teng L, Opanasopit P, Lee RJ. Nonionic surfactant vesicles for delivery of RNAi therapeutics. Nanomedicine. 2013;8(11):1865-1873.

19. Lai J, Lu Y, Yin Z, Hu F, Wu W. Pharmacokinetics and enhanced oral bioavailability in beagle dogs of cyclosporine A encapsulated in glyceryl monooleate/poloxamer 407 cubic nanoparticles. Int J Nanomedicine. 2010;5(1):13-23.

20. Boyd BJ, Whittaker DV, Khoo S-M, Davey G. Hexosomes formed from glycerate surfactants - formulation as a colloidal carrier for irinotecan. Int J Pharm. 2006;318(1-2):154-162.

21. Lai J, Chen J, Lu Y, et al. Glyceryl monooleate/poloxamer 407 cubic nanoparticles as oral drug delivery systems: I. In vitro evaluation and enhanced oral bioavailability of the poorly water-soluble drug simvastatin. AAPS PharmSciTech. 2009;10(3):960-966.

22. Chen Y, Lu Y, Zhong Y, Wang Q, Wu W, Gao S. Ocular delivery of cyclosporine A based on glyceryl monooleate/poloxamer 407 liquid crystalline nanoparticles: preparation, characterization, in vitro corneal penetration and ocular irritation. $J$ Drug Target. 2012;20(10): 856-863.

23. Esposito E, Cortesi R, Drechsler M, et al. Cubosome dispersions as delivery systems for percutaneous administration of indomethacin. Pharm Res. 2005;22(12):2163-2173.

24. Thapa RK, Baskaran R, Madheswaran T, Kim JO, Yong CS, Yoo BK. In vitro release and skin permeation of tacrolimus from monooleinbased liquid crystalline nanoparticles. J Drug Deliv Sci Technol. 2012; 22(6):479-484.

25. Liu Y, Friberg SE. Role of liquid crystal in the emulsification of a gel emulsion with high internal phase fraction. J Colloid Interface Sci. 2009; 340(2):261-268. 
26. Huang X, Brazel CS. On the importance and mechanisms of burst release in matrix-controlled drug delivery systems. J Control Release. 2001; 73(2-3):121-136.

27. Siegel RA, Rathbone MJ. Overview of controlled release mechanisms. In: Siepmann J, Siegel RA, Rathbone MJ, editors. Fundamentals and Applications of Controlled Release Drug Delivery. Boston, MA: Springer US; 2011:19-44.

28. Higuchi WI. Diffusional models useful in biopharmaceutics - drug release rate processes. J Pharm Sci. 1967;56:315-324.

29. Fong W-K, Hanley T, Boyd BJ. Stimuli responsive liquid crystals provide "on-demand" drug delivery in vitro and in vivo. $J$ Control Release. 2009;135(3):218-226.

30. Zabara A, Mezzenga R. Controlling molecular transport and sustained drug release in lipid-based liquid crystalline mesophases. J Control Release. 2014;188:31-43.

31. Negrini R, Mezzenga R. pH-responsive lyotropic liquid crystals for controlled drug delivery. Langmuir. 2011;27(9):5296-5303.

32. Chen Y, Ma P, Gui S. Cubic and hexagonal liquid crystals as drug delivery systems. Biomed Res Int. 2014;2014:1-12.

33. dos Santos S, Medronho B, dos Santos T, et al. Amphiphilic Molecules in Drug Delivery Systems. In: Coelho J, editor. Drug Delivery Systems: Advanced Technologies Potentially Applicable in Personalised Treatment. Vol. 4. Advances in Predictive, Preventive and Personalized Medicine. Dordrecht: Springer Netherlands; 2013:35-85.

34. Kumar GP, Rajeshwarrao P. Nonionic surfactant vesicular systems for effective drug delivery - an overview. Acta Pharm Sin B. 2011;1(4): 208-219.

35. Arunothayanun P, Bernard MS, Craig DQM, Uchegbu IF, Florence AT. The effect of processing variables on the physical characteristics of non-ionic surfactant vesicles (niosomes) formed from a hexadecyl diglycerol ether. Int J Pharm. 2000;201(1):7-14.

36. Lara MG, Bentley MVLB, Collett JH. In vitro drug release mechanism and drug loading studies of cubic phase gels. Int J Pharm. 2005; 293(1-2):241-250.

37. Muller-Goymann CC. Physicochemical characterization of colloidal drug delivery systems such as reverse micelles, vesicles, liquid crystals and nanoparticles for topical administration. Eur J Pharm Biopharm. 2004;58(2):343-356.

38. Chang CM, Bodmeier R. Low viscosity monoglyceride-based drug delivery systems transforming into a highly viscous cubic phase. Int $J$ Pharm. 1998;173(1-2):51-60.

39. Eccleston GM, Beattie L. Microstructural changes during the storage of systems containing cetostearyl alcohol/polyoxyethylene alkyl ether surfactants. Drug Dev Ind Pharm. 1988;14(15-17):2499-2518.

40. Dong Y-D, Boyd BJ. Applications of X-ray scattering in pharmaceutical science. Int J Pharm. 2011;417(1-2):101-111.

41. Rizwan SB, Dong YD, Boyd BJ, Rades T, Hook S. Characterisation of bicontinuous cubic liquid crystalline systems of phytantriol and water using cryo field emission scanning electron microscopy (cryo FESEM) Micron. 2007;38(5):478-485.

42. Nakano M, Sugita A, Matsuoka H, Handa T. Small-angle X-ray scattering and 13C NMR investigation on the internal structure of "Cubosomes". Langmuir. 2001;17(13):3917-3922.

43. Libster D, Aserin A, Wachtel E, Shoham G, Garti N. An HII liquid crystal-based delivery system for cyclosporin A: physical characterization. J Colloid Interface Sci. 2007;308(2):514-524.

44. Rosevear FB. Liquid crystals: the mesomorphic phases of surfactant compositions. J Soc Cosmet Chem. 1968;19:581-594.

45. Boyd BJ, Whittaker DV, Khoo S-M, Davey G. Lyotropic liquid crystalline phases formed from glycerate surfactants as sustained release drug delivery systems. Int J Pharm. 2006;309(1-2):218-226.

46. Bender J, Simonsson C, Smedh M, Engstrom S, Ericson MB. Lipid cubic phases in topical drug delivery: visualization of skin distribution using two-photon microscopy. J Control Release. 2008;129(3):163-169.

47. Bender J, Jarvoll P, Nyden M, Engstrom S. Structure and dynamics of a sponge phase in the methyl delta-aminolevulinate/monoolein/water/propylene glycol system. J Colloid Interface Sci. 2008;317(2):577-584.
48. Boyd BJ, Khoo S-M, Whittaker DV, Davey G, Porter CJH. A lipid-based liquid crystalline matrix that provides sustained release and enhanced oral bioavailability for a model poorly water soluble drug in rats. Int $J$ Pharm. 2007;340(1-2):52-60.

49. Sikora A. Utilization of various atomic force microscopy techniques in investigation of liquid crystal compounds. In: Iwan A, Schab-Balcerzak E, editors. Liquid Crystalline Organic Compounds and Polymers as Materials of the XXI Century: From Synthesis to Applications. Trivandrum: Transworld Research Network; 2011:191-219.

50. Neto C, Aloisi G, Baglioni P. Imaging soft matter with the atomic force microscope: cubosomes and hexosomes. JPhys Chem B. 1999;103(19): 3896-3899.

51. Omray LK. Liquid crystals as novel vesicular delivery system: a review. Curr Trends Technol Sci. 2013;2(6):347-353.

52. Rajabalaya R, David SR, Chellian J, Xin Yun G, Chakravarthi S. Transdermal delivery of oxybutynin chloride proniosomal gels for the treatment of overactive bladder. Drug Deliv. 2016;23(5):1578-1587.

53. Jin X, Zhang Z, Sun E, et al. Enhanced oral absorption of 20(S)protopanaxadiol by self-assembled liquid crystalline nanoparticles containing piperine: in vitro and in vivo studies. Int $J$ Nanomedicine. 2013;8:641-652.

54. Yang Z, Tan Y, Chen M, et al. Development of amphotericin B-loaded cubosomes through the SolEmuls technology for enhancing the oral bioavailability. AAPS PharmSciTech. 2012;13(4):1483-1491.

55. Porter CJH, Pouton CW, Cuine JF, Charman WN. Enhancing intestinal drug solubilisation using lipid-based delivery systems. Adv Drug Deliv Rev. 2008;60(6):673-691.

56. Kossena GA, Charman WN, Wilson CG, et al. Low dose lipid formulations: effects on gastric emptying and biliary secretion. Pharm Res. 2007;24(11):2084-2096.

57. Elnaggar YSR. Multifaceted applications of bile salts in pharmacy: an emphasis on nanomedicine. Int J Nanomedicine. 2015;10: 3955-3971.

58. Borgström B, Patton JS. Luminal events in gastrointestinal lipid digestion. Comprehensive Physiology. Hoboken, NJ: John Wiley \& Sons, Inc.; 2011:475-504.

59. Porter CJH, Trevaskis NL, Charman WN. Lipids and lipid-based formulations: optimizing the oral delivery of lipophilic drugs. Nat Rev Drug Discov. 2007;6(3):231-248.

60. McClements DJ. Encapsulation, protection, and release of hydrophilic active components: potential and limitations of colloidal delivery systems. Adv Colloid Interface Sci. 2015;219:27-53.

61. Sagalowicz L, Leser ME, Watzke HJ, Michel M. Monoglyceride selfassembly structures as delivery vehicles. Trends Food Sci Technol. 2006;17(5):204-214.

62. Lee KWY, Nguyen TH, Hanley T, Boyd BJ. Nanostructure of liquid crystalline matrix determines in vitro sustained release and in vivo oral absorption kinetics for hydrophilic model drugs. Int J Pharm. 2009; 365(1-2):190-199.

63. Sallam AS, Khalil E, Ibrahim H, Freij I. Formulation of an oral dosage form utilizing the properties of cubic liquid crystalline phases of glyceryl monooleate. Eur J Pharm Biopharm. 2002;53(3): 343-352.

64. Linkeviciute A, Misiunas A, Naujalis E, Barauskas J. Preparation and characterization of quercetin-loaded lipid liquid crystalline systems. Colloids Surf B Biointerfaces. 2015;128:296-303.

65. Borgheti-Cardoso LN, Depieri LV, Kooijmans SAA, et al. An in situ gelling liquid crystalline system based on monoglycerides and polyethylenimine for local delivery of siRNAs. Eur J Pharm Sci. 2015;74: 103-117.

66. Qin L, Mei L, Shan Z, et al. Phytantriol based liquid crystal provide sustained release of anticancer drug as a novel embolic agent. Drug Dev Ind Pharm. 2015;3:1-10.

67. Souza C, Watanabe E, Borgheti-Cardoso LN, De Abreu Fantini MC, Lara MG. Mucoadhesive system formed by liquid crystals for buccal administration of poly(hexamethylene biguanide) hydrochloride. J Pharm Sci. 2014;103(12):3914-3923. 
68. Zhang J, Lv H, Jiang K, Gao Y. Enhanced bioavailability after oral and pulmonary administration of baicalein nanocrystal. Int J Pharm. 2011;420(1):180-188.

69. Swarnakar NK, Thanki K, Jain S. Enhanced antitumor efficacy and counterfeited cardiotoxicity of combinatorial oral therapy using doxorubicinand coenzyme Q10-liquid crystalline nanoparticles in comparison with intravenous adriamycin. Nanomedicine. 2014;10(6):1231-1241.

70. Okawara M, Hashimoto F, Todo H, Sugibayashi K, Tokudome Y. Effect of liquid crystals with cyclodextrin on the bioavailability of a poorly water-soluble compound, diosgenin, after its oral administration to rats. Int J Pharm. 2014;472(1-2):257-261.

71. Parmar R, Misra R, Mohanty S. In vitro controlled release of Rifampicin through liquid-crystalline folate nanoparticles. Colloids Surf B Biointerfaces. 2015;129:198-205.

72. Pham AC, Nguyen T-H, Nowell CJ, Graham B, Boyd BJ. Examining the gastrointestinal transit of lipid-based liquid crystalline systems using whole-animal imaging. Drug Deliv Transl Res. 2015;5(6):1-9.

73. Bhatt AB, Barnes TJ, Prestidge CA. Silica nanoparticle stabilization of liquid crystalline lipid dispersions: impact on enzymatic digestion and drug solubilization. Curr Drug Deliv. 2015;12(1):47-55.

74. Nguyen TH, Hanley T, Porter CJH, Boyd BJ. Nanostructured reverse hexagonal liquid crystals sustain plasma concentrations for a poorly water-soluble drug after oral administration. Drug Deliv Transl Res. 2011;1(6):429-438.

75. Bisset NB, Boyd BJ, Dong Y-D. Tailoring liquid crystalline lipid nanomaterials for controlled release of macromolecules. Int J Pharm. 2015;495(1):241-248.

76. Estracanholli EA, Praça FSG, Cintra AB, Pierre MBR, Lara MG. Liquid crystalline systems for transdermal delivery of celecoxib: In Vitro drug release and skin permeation studies. AAPS PharmSciTech. 2014;15(6):1468-1475.

77. Lopes LB, Speretta FFF, Bentley MVLB. Enhancement of skin penetration of vitamin K using monoolein-based liquid crystalline systems. Eur J Pharm Sci. 2007;32(3):209-215.

78. Lopes LB, Lopes JLC, Oliveira DCR, et al. Liquid crystalline phases of monoolein and water for topical delivery of cyclosporin A: characterization and study of in vitro and in vivo delivery. Eur J Pharm Biopharm. 2006;63(2):146-155.

79. Uchino T, Matsumoto Y, Murata A, Oka T, Miyazaki Y, Kagawa Y. Transdermal delivery of flurbiprofen from surfactant-based vesicles: particle characterization and the effect of water on in vitro transport. Int J Pharm. 2014;464(1-2):75-84.

80. Otto A, du Plessis J, Wiechers JW. Formulation effects of topical emulsions on transdermal and dermal delivery. Int J Cosmet Sci. 2009; 31(1):1-19.

81. Lee BJ, Ryeol D, Cho N, et al. Efficient dermal delivery of retinyl palmitate: progressive polarimetry and Raman spectroscopy to evaluate the structure and efficacy. Eur J Pharm Sci. 2015;78:111-120.
82. Westesen K, Bunjes H, Hammer G, Siekmann B. Novel colloidal drug delivery systems. PDA J Pharm Sci Technol. 2001;55(4):240-247.

83. Chaiyana $\mathrm{W}$, Rades T, Okonogi S. Characterization and in vitro permeation study of microemulsions and liquid crystalline systems containing the anticholinesterase alkaloidal extract from Tabernaemontana divaricata. Int J Pharm. 2013;452(1-2):201-210.

84. Rarokar NR, Saoji SD, Raut NA, Taksande JB, Khedekar PB, Dave VS. Nanostructured cubosomes in a thermoresponsive depot system: an alternative approach for the controlled delivery of docetaxel. AAPS PharmSciTech. 2015;17(2):1-10.

85. Zhang Y, Zhang K, Guo T, Li Y, Zhu C, Feng N. Transdermal baicalin delivery using diethylene glycol monoethyl ether-mediated cubic phase gel. Int J Pharm. 2014;479(1):219-226.

86. Oyafuso MH, Carvalho FC, Chiavacci LA, Gremiao MPD, Chorilli M. Design and characterization of silicone and surfactant based systems for topical drug delivery. J Nanosci Nanotechnol. 2015;15(1):817-826.

87. Nesseem DI. Formulation and evaluation of itraconazole via liquid crystal for topical delivery system. J Pharm Biomed Anal. 2001;26(3): 387-399.

88. Singh VK, Pal K, Banerjee I, Pramanik K, Anis A, Al-Zahrani SM. Novel organogel based lyotropic liquid crystal physical gels for controlled delivery applications. Eur Polym J. 2015;68:326-337.

89. Angelov B, Angelova A, Garamus VM, et al. Small-angle neutron and X-ray scattering from amphiphilic stimuli-responsive diamondtype bicontinuous cubic phase. J Am Chem Soc. 2007;129(44): 13474-13479.

90. Zhang W, Liu L. Study on the formation and properties of liquid crystal emulsion in cosmetic. J Cosmet Dermatological Sci Appl. 2013;3(2): 139-144.

91. Dong Y, Larson I, Hanley T, Boyd BJ. Bulk and dispersed aqueous phase behavior of phytantriol: effect of vitamin E acetate and F127 polymer on liquid crystal nanostructure. Langmuir. 2006;22(23):9512-9518.

92. Clogston J, Craciun G, Hart DJ, Caffrey M. Controlling release from the lipidic cubic phase by selective alkylation. J Control Release. 2005; 102(2):441-461.

93. Narang AS, Delmarre D, Gao D. Stable drug encapsulation in micelles and microemulsions. Int J Pharm. 2007;345(1-2):9-25.

94. Nguyen TH, Hanley T, Porter CJH, Boyd BJ. Nanostructured liquid crystalline particles provide long duration sustained-release effect for a poorly water soluble drug after oral administration. J Control Release. 2011;153(2):180-186.

95. Gosenca M, Bester-Rogac M, Gasperlin M. Lecithin based lamellar liquid crystals as a physiologically acceptable dermal delivery system for ascorbyl palmitate. Eur J Pharm Sci. 2013;50(1):114-122.

96. Luo M, Shen Q, Chen J. Transdermal delivery of paeonol using cubic gel and microemulsion gel. Int J Nanomedicine. 2011;6:1603-1610.
Drug Design, Development and Therapy

\section{Publish your work in this journal}

Drug Design, Development and Therapy is an international, peerreviewed open-access journal that spans the spectrum of drug design and development through to clinical applications. Clinical outcomes, patient safety, and programs for the development and effective, safe, and sustained use of medicines are the features of the journal, which

\section{Dovepress}

has also been accepted for indexing on PubMed Central. The manuscript management system is completely online and includes a very quick and fair peer-review system, which is all easy to use. Visit http://www.dovepress.com/testimonials.php to read real quotes from published authors. 